\title{
Editorial
}

\section{Challenges Facing Pediatric Dentistry Diplomates and the American Board of Pediatric Dentistry: Setting the Bar for Minimal Competency vs. Excellence}

\author{
John E. Nathan, DDS, MDS, FAAPD, MASDC* \\ Department of Pediatric Dentistry, University of Alabama, Birmingham, and Case Western Reserve University, Cleveland, USA \\ "Corresponding author \\ John E. Nathan, DDS, MDS, FAAPD, MASDC \\ Adjunct Professor, Department of Pediatric Dentistry, University of Alabama, Birmingham, and Case Western Reserve University, Cleveland, USA; \\ E-mail: jnathandds@gmail.com
}

\section{Article information}

Received: March 21 ${ }^{\text {st }}, 2019$; Accepted: March 22 ${ }^{\text {nd }}, 2019$; Published: March 26 ${ }^{\text {th }}, 2019$

\section{Cite this article}

Nathan JE. Challenges facing pediatric dentistry diplomates and the american board of pediatric dentistry: Setting the bar for minimal competency vs. excellence. Pediatr Neonatal Nurs Open J. 2019; 6(I): e5-e7. doi: 10.17I40/PNNOJ-6-e009

\section{ABSTRACT}

Over the past two decades, the American Board of Pediatric Dentistry (ABPD) has admirably and conscientiously grappled with the dilemma of how to maintain the bar of clinical competency while vastly enhancing its membership numbers to pursue board certification. This editorial seeks to discuss the changes which have occurred historically that has impacted on the nature and quality of the examination process that is used to determine what constitutes a level of competency for pediatric dentists in the U.S. For matters of practicality and consistency throughout various disciplines within medicine and dentistry, perceptions have increasingly moved in the direction that a degree of competency be defined for which the vast majority of providers potentially qualify. While it would be optimal to set the bar for achievement at the level of excellence, doing such excludes the bulk of practitioners as previously existed in health care during the previous half-century. The need to redefine what sufficiently constitutes merely an acceptable level of comprehension has become the rule rather than the exception by today's standards. Recognition of the achievement of excellence, while beyond the purview of certifying boards, might still best be re-examined, re-designed and awarded to the benefit of the practitioners and public these boards are intended to endorse and serve. The fact of the matter is that satisfactory completion of current board certification does not carry the weight or prestige it once represented. Certification boards might do well to reassess or reframe the reward of Diplomate status on the basis of the achievement of excellence $v s$. adequacy that is measured.

$\mathrm{P}$ rior to 2002, the process of board certification in pediatric dentistry involved a rigorous four-part format which required a minimum of three to four years to complete. The initial or eligibility part began with an all-day written examination based and referenced from a well-conceived and lengthy 200 article reading list of classical and contemporary literature spanning all relevant and related areas of pediatric dentistry. Upon completion of part 1, applicants were considered eligible to participate in the next three sections. Part 2 included freelance style oral examination by two peers over approximately one hour on any conceivable subject(s). Part 3 consisted of submission of 5-6 documented cases involving specific but commonly seen clinical entities requiring pre-treatment, immediate post-treatment, and follow-up results to demonstrate and document ones diagnostic and clinical skills. The final section, Part 4, included an all-day site visit to illustrate clinician judgment, hands-on skills and general decorum and staff interaction in one's clinical setting. This author completed the certification process in 1983, a time when approximately $15 \%$ of the membership the American Academy of Pediatric Dentistry (AAPD) had sought and completed certification. The process of board certification between specialties at this time was similar with some minor degree of variation and expectation while the focus was sustained toward the pursuit of excellence. At that time a similarly small percentage pursued certification in orthodontics. An image of elite status prevailed that readily acknowledged that those having completed the process were considered among the brightest and most talented in their field.

Many if not the vast majority of specialists elected not to pursue certification because of its degree of difficulty, effort de- 
manded, costs involved, and minimal impact on private practice reward. During this period, practitioners were not penalized for not having secured certification other than to refrain from the mention of having secured diplomate status to their clientele. During the latter, 1980's and early 1990's hospital staff bylaws began moving in the direction that if a clinical specialty participated in an American Board, board certification was required by the time of reappointment to the medical staff. Because hospital dentistry is an active component of pediatric dentistry, the ability to admit patients for treatment under anesthesia served as a significant incentive for pediatric dentists to seek board certification. This became a timely opportunity for reassessment of the ABPD examination process to explore what might be needed to encourage interest and expand membership to seek certification. Herein was a crossroad which challenged the mindset of either a pursuit of excellence, hence elite recognition of clinician skills and competency $v s$. a significant lowering of the bar to what constituted a minimum standard that most clinicians could satisfy. To preserve an ability to make use of general anesthesia for their patients, clinicians without hospital appointment or privileges, make use of an itinerant anesthesiologist in an office setting, or use of freestanding surgical centers where costs are less prohibitive and care was accessible to those with inadequate or no medical insurance coverage.

During a short interim between major changes in the two formats, applicants had the choice of taking the original format or the much-abbreviated revision. It became clear that advancing the numbers in pursuit of certification would result and be attractive only in the direction of the abbreviated format. Hundreds of pediatric dentists now appear annually to pursue board certification unlike before with the more demanding and lengthy four-part format.

It became logical that exploration to re-define the goals and objectives of being certified to identify minimally competent levels $v s$. elite or extraordinary status has evolved to the present day examination process, which is substantially abbreviated in comparison to its earlier form. Reports indicate that substantial increases in membership receiving Diplomate status have resulted from the substantial reductions in the length and demands of the new format. If the goal was to redefine what simply constituted the minimum level of clinician sophistication and competency, which could be argued better and more fairly represents the present-day standard for a given discipline, then little opposition to a divestiture from elite recognition should result. That said, however, the bottom line has in actuality become the pursuit of a minimal level of expectation which depending on one's perspective is now considered both acceptable and reasonable. For those having completed the more demanding four-part format, however, impressions conceivably and justifiably may lean in the direction that the level of excellence achieved from more rigorous and demanding preparation should warrant some tangible form of greater distinction if not recognition than the current certification status.

This in no way should imply the ABPD has been anything short of diligent in its commitment and dedication to creating and continually updating the certification process. Settling for the status quo is not among its mission. The ABPD, like other specialties in both medicine and dentistry, has in a subtle way gone that direction by further changes that have instituted a Re-Certification examination. Through such, those having completed the more demanding previous format are exempt from having to complete a recertification examination. These Diplomates have been granted "unlimited" certification status. Arguments both in support (or the lack thereof) for the need for re-certification exist. The implementation of a Re-Certification examination is not uncommon today among many medical and dental disciplines; all fields undergo dynamic changes and additions of new knowledge over time. Recertification, therefore, appeals as a desirable mechanism by which verification that clinicians have upgraded and further advanced their own knowledge base can be considered useful and appropriate. Herein lies a fundamental discrepancy between clinicians, once masters of their craft, who conceivably practice within the same framework and parameters over a lengthy career without fervent intent and desire to update their skills. For others, however, one might argue that recertification can be considered unnecessary in light of the existence of licensure demands in all states for mandatory continuing education. Alternatively, movement in this direction only serves to foster a greater pursuit of excellence and raise the proverbial bar of competency. Re-Certification today comprises a 50 question written multiple choices, open book examination. Successful Recertification requires a minimum of $80 \%$ correct responses. This author would hypothetically argue that for those having completed the original four-part format, dedication to lifelong learning likely more accurately characterizes their recognition of the need to stay current and master new and developing knowledge. Challenge to the validity and need for a fifty question multiple choice examination to serve as validation and verification of one's level of current competency is at best suspicious. One conceivable and fiscal argument for recertification might be that having such exposes a large number of candidates to expanded revenues for the board.

This author would encourage the ABPD to explore or survey the viewpoint of its members relative to their own perceptions of the merit and personal accomplishment from participation in the certification process, be it from the original format to the current format. I further encourage the board to consider a further delineation in Diplomate Status for those who have completed the original far more demanding format as compared to the current abbreviated format. Call it Diplomate "Master," or Diplomate $(*)$, or some appropriate symbol, the distinction seems warranted. Recognition of minimal levels of competency $v s$. the highest level of the pursuit of the highest level of excellence should accompany this noble and gratifying achievement. On the other hand, the American Board should be commended for their fervent and dedicated efforts to update its examinations, using every means within its power to demonstrate validity be it through psychometric evaluation and contemporary assessment criteria. Re-assessment to include classical as well as contemporary literature, inclusive of evidence-based studies as available, across various areas of mainstream topics seems warranted. Expansion of questioning and exploration in areas of safe and effective pediatric sedation, recognition and management of adverse reactions and medical emergencies, pediatrics, orthodontics and fundamental aspects of growth and development, assessment of the mixed dentition and 
rationale for when space maintenance is warranted are among useful areas for inclusion. Having from an academic and private practice environment met and interviewed numerous potential applicants for private practice settings who have completed certification with the abbreviated format, and postgraduate students over the past several decades, these areas constitute in this author's opinion, areas of weakness in need of attention. In final analyses, these aspects will remain amongst the challenges of advanced training in pediatric dentistry and the American Board whose responsibilities fall in establishing standards for the field and setting the bar for the pursuit of minimal competency $v$ s. excellence.

\section{ACKNOWLEDGEMENTS}

The opinions expressed in this editorial are those of the author and not in any way to be construed as those representing the opinions of the ABPD or its board of Directors. 